\title{
Adsorption of Benzene by "Green" functionalization of Montmorillonite
}

\author{
Hirra Anjum ${ }^{1}$, Mansoor Abdullah Obaid ${ }^{1}$, Muhammad Yasir Shamim ${ }^{1}$, Thanabalan Murugesan ${ }^{*}$ \\ ${ }^{1}$ Department of Chemical Engineering, Universiti Teknologi PETRONAS, Bandar Seri Iskandar 32610, Perak, Malaysia.
}

\begin{abstract}
The capacity of organically modified Montmorillonite (MMT) clay to adsorb nonpolar organic compound (benzene) in an aqueous solution, was investigated under the batch process. MMT was pretreated (centrifuged) and then functionalized with green intercalating agent i.e. 1hexyl-3-methyl imadazolium chloride [HMim][Cl]. The characterization through Fourir Transoform Infrared Spectroscopy (FTIR), Differential Scanning Calorimeter (DSC) and Field Emission Scanning Electron Microscope (FE-SEM) confirmed the presence of the oxygen containing functional groups, changes in melting point and variation in the morphological properties. The governing parameters for the sorption of benzene such as the effect of contact time, $\mathrm{pH}$, adsorbent dose and rotation were studied. The kinetic data conformed to pseuodo $2^{\text {nd }}$ order kinetic model and the isotherm experimental data were a better fit to Langmuir model with maximum adsorption capacity of $588.23 \mathrm{mg} / \mathrm{g}$ under experimental conditions. Overall, MMT intercalated with 1-hexyl-3methyl imadazolium chloride is a promising environmental friendly adsorbent for the abatement of benzene in an aqueous solution.
\end{abstract}

\section{Introduction}

In the last few years, the application of energy and power sources has been increased in the world. Nowadays, the main sources of environmental pollutants are industrialization, civilization, agricultural activities and other environmental changes. Large quantities of dangerous pollutants are emitted by these sources. Benzene has been consigned as one of the dangerous hazardous material. A severe exposure by inhalation or ingestion can be lethal. The maximum allowable concentration of benzene in drinking water is $0.01 \mathrm{mg} / \mathrm{L}$. Therefore, many conventional desalination technologies have been employed for wastewater treatment. But, excessive chemical usage and sludge accumulation prove them to be ineffective methodologies. However, adsorption is found to be superior in performance in terms of simple design, ease of operation and nontoxic adsorbent utilization. Furthermore, availability, costeffectiveness and environmental friendly nature are the major factors in the selection of green adsorbent [1].

The crystalline clay minerals are the fundamental constituents of the phyllosilicates. Their network structure encapsulates two, three or four individual sheets. These sheets are usually comprised of tetrahedral $\left(\mathrm{SiO}_{4}\right)^{4-}$ and octahedral $\left(\mathrm{AlO}_{3}(\mathrm{OH})_{3}\right)^{6-}$ sheets. Furthermore, the silicate layers are capable of arranging themselves in the form of stacks having a regular van der Waals gap (interlayer) between them. These layers assimilate small cationic structures and some of the peaks are connected to protons $(\mathrm{OH})$ because they are

\footnotetext{
* Corresponding author: murugesan@utp.edu.my
}

occupied by oxygen [2]. However, isomorphous substitution within the sheets is the only factor responsible for the presence of exchangeable cations. These ionic substitutions are managed by the environmental chemistry and the reaction kinetics during the generation of clay minerals [3-5]. Thus, the characteristics that make it a suitable adsorbent for several contaminants abatement from polluted water are the surface electric charge, internal porosity, surface functional groups and predominantly, exchangeable cations inside crystal lattice. The pre-eminent consumers of these moieties are petroleum industries, steel mills, paint and varnish industries etc. [6].

However, the hydrophilic clays are proved to be ineffective adsorbent for abatement of aromatic compounds because of very low sorption capacity and selectivity. Probably, the water cluster formation is held responsible for minimization of adsorption efficiency. Taking into account these considerations, various approaches i.e., the introduction of exchangeable cations, pillarization [7], thermal treatment $[8]$ and acid activation [9] have been employed to improve the configuration, conformation and framework of clay for uptaking the organic pollutants.

On the other hand, the uptake of organic/inorganic pollutant via clay adsorbent is a complex phenomenon involving more than one mechanisms. These techniques inculcate surface adsorption [10], ion exchange [11], ligand exchange [12], surface partition [13], surface precipitation [14] and structural incorporation [15]. 
Furthermore, structure, molecular size, partition coefficient, hydrophobicity, polarity [16] are major responsible factors for the adsorption affinity interactions of benzene for clay adsorbent [17].

Organoclay minerals prepared with small groups tend to sorb targetted pollutants by surface adsorption because small molecules occupy outer energetic sites immediately and very few adsorb inside the interpenetrating network structure. However, clay adsorbent with large electron donor/acceptor group tends to remove the hazardous material by partitioning as the big structures occupy a large number of active sites of the interlamellar spaces of the clay [18]. It was approved by Jaynes et al. (1999) in which they concluded that SWy-TMA and SWy-Adam (small groups) removed benzene through partitioning process while SWyHDTMA (bulky group) followed the surface adsorption path [19].Since the ordered structure with large molecular weight lead towards higher sorption capacity. While working with bentonite clays, Bertagnolli et al. (2012) deduced that organophilization altered the hydrophilic character of clay structure into hydrophobic channels by replacing inorganic cations with organic cations that in turn, caused basal spacing to increase 15.2 to $23.9 \AA$. This factor was solely, responsible for the strong interactions between BTX and organoclay framework [20]. Additionally, Koh et al. (2001) proved that the bulky groups (BDTDA) of surfactants attached to the clay minerals, cause the multiple interlayer adsorption as compared to the smaller groups (BTMA) that show the reluctance for second interlayer sorption for benzene and toluene [21]. More importantly, steric constrains of cations attached to the clay framework also affects the overall uptake of organic compounds. The study revealed that the cations used in organoclays may accommodate BTX either in parallel or tilted orientation with clay surface. The probability of BTX to be arranged in either manner depends upon the size distribution of free area. Thus, the smaller active sites favor, the tilted orientation while, as the size increases from benzene to xylene, cationic molecules are forced to arrange in parallel manner relative to clay layers [22].

However, Carvalho et al. (2012) endorsed that polarity effect of BTEX compounds is mainly due to the inductive effect (presence of delocalized $\pi$-electrons in ring structure) stimulated by methyl and ethyl groups. Moreover, adsorption isotherm data suggested that the heterogeneous sorption sites exist on the surface of semectite clay[23]. Additionally, the interaction between water molecules and sorbent with oxygen bearing functionl groups create some competition in sorption of organic compounds by establishiing hydrogen bond interactions. This effect is observed more in the large nonpolar molecules. While, Qu et al. (2009) summarized that cross-sectional area, critical volume, polarizability and enthalpy of vaporization are the influencing parameters for adsorption capacity. they elucidated that the dispersion interactions of BTEX compounds represent $\pi-\pi$ electron interactions (weak hydrogen bond) while acetone comes up with dipole induced dipole forces (medium hydrogen bond).thus, because of stronger intermolecular interaction, acetone was considered to posess high adsorption affinity than that of BTEX [24]. Nevertheless, $\mathrm{pH}$ of the solution and humidity are not the controlling factor in the adsorption of aromatic compounds through organo-clays. Moreover, the controlling reagents to adjust the $\mathrm{pH}$, cause the structural changes from porous bed to mud like structure in organo-clay. Similarly, Nourmoradi et al. (2012) reported the same results for removal of BTEX by modified MMT. They found that the increment in $\mathrm{pH}$ values from 4 to 12 does not have the potential influence on the removal capacity. This depicts that within solution range, modified MMT has high solubility [25]. In the present study, the sorption efficiency of economically viable and green (eco-friendly) MMT was evaluated for the removal of benzene in an aqeous solution. The equilibrium isotherms and kinetics adsorption models were also investigated.

\section{Materials \& Methods}

\subsection{Materials}

Montmorillonite (MMT-K10, $250 \mathrm{~m}^{2} / \mathrm{g}$ ) and 1-hexyl-3methylimadazolium chloride $(\geq 97 \%$, MW : 202.72) were purchased from Sigma Aldrich.

\subsection{Methodology}

$10 \mathrm{~g}$ of MMT was dissolved in 1 Litre deionized water. The suspension was stirred with a mechanical stirrer at $600 \mathrm{rpm}$ for $24 \mathrm{~h}$. The solution centrifuges at $4000 \mathrm{rpm}$ for 15 minutes, consequently the impurities such as quarts were at the bottom of the centrifuge tube. Then, MMT dried at $110^{\circ} \mathrm{C}$ for $24 \mathrm{~h}$ and crushed to $124 \mu \mathrm{m}$ particles. After the pre-treatment step, $4 \mathrm{~g}$ MMT and 40 $\mathrm{ml}$ of $100 \mathrm{mmol} / \mathrm{L}$ 1-hexyl-3-methyl imadazolium chloride were mixed. The mixture was agitated at $150 \mathrm{rpm}$ for one day at room temperature and centrifuged for half an hour. It was then dried in an oven at $40^{\circ} \mathrm{C}$ for $8 \mathrm{~h}$. The schematic diagram of the surface modification of MMT is represented in Figure1.

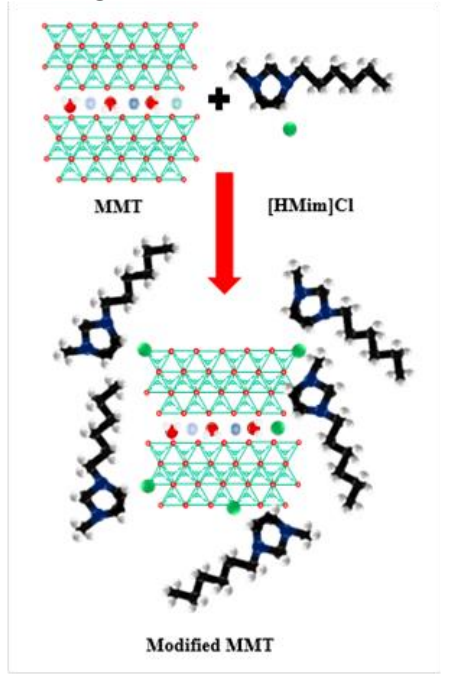

Fig.1. The schematic diagram of the surface modification of MMT. 


\section{Results and discussion}

\subsection{Infrared analysis}

FTIR analysis of MMT before and after the modification was performed to identify the functional groups over the surface of clay in Figure 2. Pure MMT has only significant peak confirming functional groups to abate benzene is at $3490 \mathrm{~cm}^{-1}$. On the other hand, modified MMT confirms the presence of oxygen containing functional groups at $3200 \mathrm{~cm}^{-1}, 3000 \mathrm{~cm}^{-1}, \mathrm{C}-\mathrm{H}$ stretch at $2892 \mathrm{~cm}^{-1}, \mathrm{C}=\mathrm{C}$ stretching vibration at $1550 \mathrm{~cm}^{-1}, 1440 \mathrm{~cm}^{-1}$ that can not be found in Pure MMT.

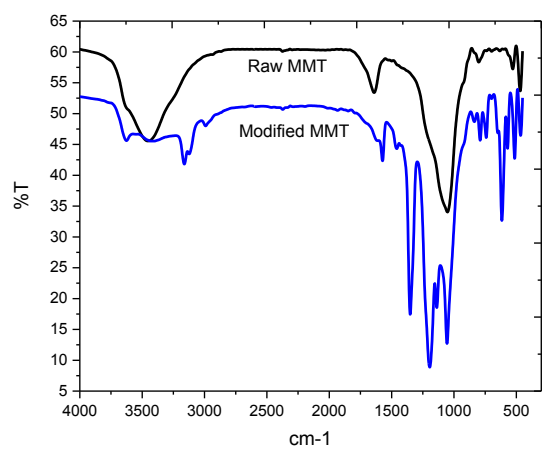

Fig. 2. IR spectra of raw and modified MMT.

\subsection{DSC analysis}

The thermal behavior of MMT before and after the surface modification is measured by DSC Q2000 V24.11 build 124, with a heating and cooling ramp of $10 \mathrm{~K} / \mathrm{min}$ from $0^{\circ} \mathrm{C}$ to $300^{\circ} \mathrm{C}$ under nitrogen flow and the values were recorded during the second heating scan. The endothermic peak which corresponds to the melting temperature is presented at $120^{\circ} \mathrm{C}$ for pure MMT whereas it goes down to $99.6{ }^{\circ} \mathrm{C}$ for

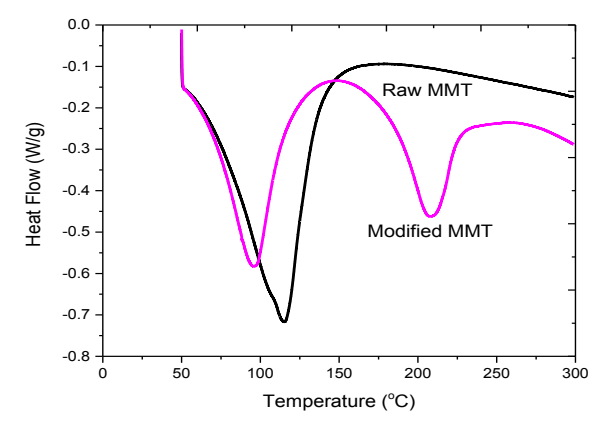

Fig. 3. DSC results of raw and modified MMT.

functionalized MMT. The crystallization temperature goes down from $175^{\circ} \mathrm{C}$ to $150^{\circ} \mathrm{C}$ which confirms the presence of functional group over the surface of pure MMT.

\subsection{Scanning electron microscope analysis}

Figure 4 shows the SEM images of structure of the MMT before and after the surface modification. Figure $3 \mathrm{a}$ depicts the surface roughness before the modification step. However, after modification, the surface exfoliation takes place as well as agglomeration is reduced due to the presence of functional groups that have an affinity for benzene as shown in Figure $4 b$.
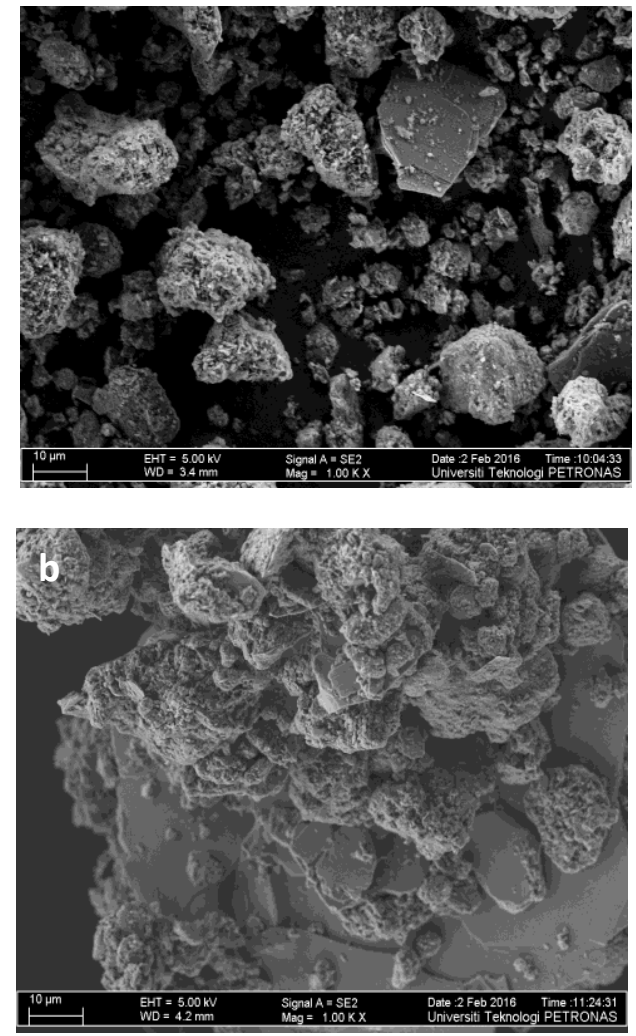

Fig. 4. FE-SEM results of a) raw and b) modified MMT.

However, the EDS spectra shows that the oxygen content has been increased after surface modification which consequently improve the sorption capacity of MMT.

\subsection{Kinetic experiments}

\subsubsection{Effect of contact time}

In order to design the batch exeriments the determination of sorption rate is the mandatory requirement. The effect of contact time on the adsorption of benzene was investigated. Figure 5 shows the amount of benzene removed by the modified MMT with respect to time. It can be clearly seen that adsorption rate initially increased rapidly, and the optimal removal efficiency was reached within about $70 \mathrm{~min}$. the adsorption capacity is recorded to be $179.63 \mathrm{mg} / \mathrm{g}$. Further increase in contact time did not manifest significant change in equilibrium concentration. 


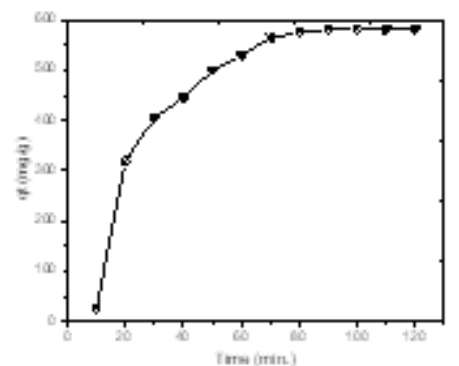

Fig.5. Effect of contact time on the adsorption of benzene.

The absorption rate decreases after equilibrium, which may be due to the fact that all the high energy sites are occupied and ions face difficulty in finding active vacant pores. This decreases the sorption capacity, which is because of the process of Intraparticle diffusion. Since, after the transfer of the ions to the surface this process occurs in the second stage of adsorption which is very slow and ultimately create repulsive forces between bulk phase and solute [26].

\subsubsection{Effect of $\mathrm{pH}$}

The $\mathrm{pH}$ of a solution is an important factor in the adsorption process because it causes protonantion or deprotonation effect. Commonly, at extremely acidic and basic conditions the adsorption capacity comes out to be very low. It is because of layophobic behavior of adsorbent. Furthermore, at extreme acidic $\mathrm{pH}$, the surface functional groups of adsorbent tend to compete with $\mathrm{H}^{+}$ions in background solution. Similarly, $\mathrm{H}_{3} \mathrm{O}^{+}$ ions compete with the surface charges at extreme basic conditions [27]. During this study, results revealed that the removal of benzene was strongly dependent on the $\mathrm{pH}$ of the solution. During the experimentation we varied the $\mathrm{pH}$ from 3 to 12 , and found that maximum amount of benzene is adsorbed at $\mathrm{pH} 8$.

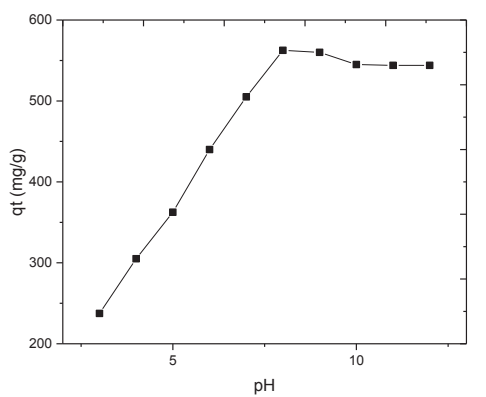

Fig. 6. Effect of $\mathrm{pH}$ on the absorption of benzene.

The amount increased rapidly with the increase in $\mathrm{pH}$ of the solution initially, and the optimal $\mathrm{pH}$ was observed at $\mathrm{pH}$ 6.5. Further increase in $\mathrm{pH}$ caused a decrease in the amount adsorbed. Thus, it can be deduced that the weak electrostatic forces of attraction exist between functionalized MMT and benzene. However, these interactions operate simultaneously. Therefore, the role of hydrogen bonding cannot be ignored as an interaction mechanism between adsorbate and adsorbent.

\subsubsection{Effect of adsorbent dose}

As adsorbent dosage increases the amount of benzene adsorbed, decreases. As adsorbent dosage increases benzene uptake decreases as shown in the Figure 7. At lower adsorbent concentration number of active sites is higher. With the increase in adsorbent dosage aggregation of particles take place, as a result benzene uptake decreases [28].

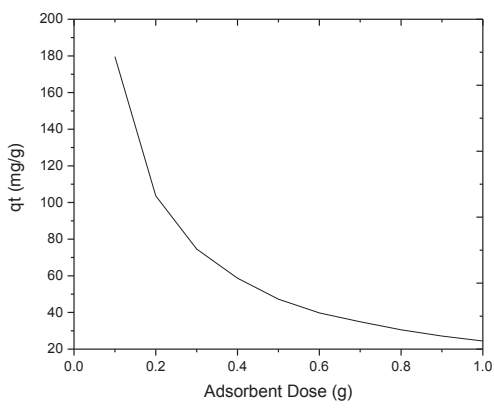

Figure 7: Effect of adsorbent dose on the adsorption of benzene.

\subsubsection{Effect of rotation}

As the rotation increases the amount of benzene removed is not much affected as presented in Figure 8 . This describes that the solution RPM does not have a direct impact on the process of adsorption.

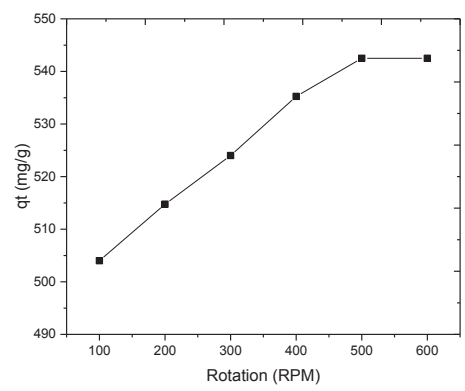

Fig. 8. Effect of rotation on the adsorption of benzene.

\subsection{Kinetic modeling}

The kinetic modelling of sorption of targetted pollutant guides in designing the adsorption experiments and helps in selection of optimum experimental conditions. These kinetic models are dependant on transfer of benzene to the surface of modified MMT, transfer from modified MMT to intraparticle diffusion, retention of ions on the surface of modfied MMT and complexation phenomena. In order to investigate the mechanism of removal of benzene from the surface of modified MMT, pseudo $1^{\text {st }}$ order, pseudo $2^{\text {nd }}$ order and intra-partile diffusion models were employed to fit the experimental data [29]. The 
kinetic study reveals that the sorption of benzene follows the pseudo $2^{\text {nd }}$ order kinetics. All three kinetic models are summerized in Table 1.

Table 1. Kinetic parameters for the adsorption of benzene by modified MMT.

\begin{tabular}{|c|c|c|}
\hline Model & Parameters & $\mathrm{R}^{2}$ \\
\hline Pseudo 1 st order & $\begin{aligned} \mathrm{q}_{\mathrm{e}} & =977.5 \mathrm{mgg}^{-1} \\
\mathrm{~K}_{1} & =0.056 \mathrm{~min}^{-1}\end{aligned}$ & 0.945 \\
\hline Pseudo $2^{\text {nd }}$ order & $\begin{aligned} \mathrm{q}_{\mathrm{e}} & =588.23 \mathrm{mgg}^{-1} \\
\mathrm{~K}_{2} & =0.0001 \mathrm{gmg}^{-1} \mathrm{~min}^{-1}\end{aligned}$ & 0.995 \\
\hline $\begin{array}{l}\text { Intraparticle } \\
\text { diffusion }\end{array}$ & $\begin{array}{l}\mathrm{K}_{\mathrm{ID}}=106.06 \mathrm{mgg}^{-1} \mathrm{~min}^{-1 /} \\
\mathrm{C}=49.34 \mathrm{gmg}^{-1} \mathrm{~min}^{-1}\end{array}$ & ${ }^{2} 0.881$ \\
\hline
\end{tabular}

\subsection{Adsorption Isotherms}

The absorption isotherms are employed to fit the experimental data in order to gain more insight of adsorption experiments. Thus, to understand the interaction between adsobent and adsorbate, isotherm study is very important. In this regard, Langmuir isotherm assumes that the surface of modified MMT is homogenous in nature and the active sites for the sorption of benzene are identical. However, Freundlich isotherm is the empiral representation and explins the heterogeneity of the sorpbent and is not restricted by the monolayer formation[30].

Table 2. Parameters of isotherm models for the adsorption of benzene by modified MMT.

\begin{tabular}{lcc}
\hline Model & Parameters & $\mathrm{R}^{2}$ \\
\hline Langmuir & $\mathrm{Q}_{\mathrm{o}}=588.23$ & 0.989 \\
& $\mathrm{~K}_{\mathrm{L}}=2.06$ & \\
Freundlich & $\mathrm{n}=0.707$ & 0.979 \\
& $\mathrm{~K}_{\mathrm{F}}=1.138$ & \\
& &
\end{tabular}

The isotherm model study summerized in Table 2, implies that Langmuir isotherm model best fits the calculated amount adsobed benzene. However, heterogeneity of the modified MMT suggests the favorable adsorption process. Hence, the values of $\mathrm{R}^{2}$ suggest that the isotherm model is adequate to describe the adsorption process well.

\subsection{Comparative analysis of sorbents}

The sorption capacities of several different types of clays (with or without modification) for the removal of benzene are summarized in Table 3 . The comparative evaluation is difficult because the sorption capacities available in literature were determined at different $\mathrm{pH}$, temperatures, adsorbent dosage and pollutant initial concentration. By keeping these caveats under consideration, selected adsorbents are compared. Although modified semectite clay removed benzene efficiently, but the introduction of ionic liquid treated MMT came up with much higher efficiency alongwith improved physicochemical properties.
Table 3. Comparative analysis for the adsorption of benzene by various types of clays.

\begin{tabular}{|c|c|c|c|}
\hline Adsorbent & Pollutant & Modifier & $\begin{array}{c}\text { Removal } \\
\text { Efficiency } \\
\text { (mg/g) }\end{array}$ \\
\hline MMT (This study) & Benzène & [HMim]Cl & 588.23 \\
\hline Bentonite [20] & Benzène & $\begin{array}{c}\text { Cetyl pyridinium } \\
\text { chloride salt }\end{array}$ & 0.22 \\
\hline MMT [21] & Benzene & $\begin{array}{c}\text { BDTDA/ BTMA/ } \\
\text { Hyamine }\end{array}$ & 28 \\
\hline Sericite mica [21] & Benzene & $\begin{array}{c}\text { BDTDA/ BTMA/ } \\
\text { Hyamine }\end{array}$ & 21 \\
\hline $\begin{array}{c}\text { Smectite [22] } \\
\text { Semectite } \\
\text { organoclay } \\
\text { [23] }\end{array}$ & Benzene & BMA & 160 \\
\hline $\begin{array}{c}\text { Na-MMT [25] } \\
\text { K-10 MMT [31] }\end{array}$ & Benzene & BDTMA chloride & 2.04 \\
\hline Na-MMT [31] & Benzene & PEG & 3.58 \\
\hline $\begin{array}{c}\text { Wyoming } \\
\text { montmorillonite } \\
\text { [31] }\end{array}$ & Benzene & HDTMA & - \\
\hline Bentonite [32] & Benzene & Raw & 0.28 \\
\hline
\end{tabular}

Some of the reported modified clays have higher surface area after modification step but the sorption efficiency for the removal of benzene turned out to be very low. This may be because of the crowded surface of MMT with oxygen containing functional groups that may cause water cluster formation. But, surface area, alone cannot be the sole governing factor in determining the sorption efficiency.

\section{Conclusion}

In the present study, montmorillonite (MMT) was modified by 1 -hexyl-3-methyleimadzolum chloride. The functionalization was approved by analytical tools i.e., FTIR, DSC and FE-SEM. However, intensive investigation of adsorption capacity through batch adsorption experiments revealed that the sorption capacity of benzene gradually increased to $581.25 \mathrm{mg} / \mathrm{g}$, which is the indication of the affinity of surface functional groups for aromatic ring structure. The adsorption equilibrium data was in good agreement with Langmuir isotherm with maximum adsorption capacity of $588.23 \mathrm{mg} / \mathrm{g}$ whereas it follows pseudo second order model for adsorption kinetic data calculations. Hence, the modified MMT is considered highly efficient for wastewater treatment.

\section{References}

1. J. i. Konta. Clay and man: clay raw materials in the service of man. Appl. Clay Sci., 10(4), 275335 (1995).

2. M. Brigatti, E. Galan, \& B. Theng. Structure and mineralogy of clay minerals. Handbook of Clay Science, Developments in Clay Science, Elsevier, 5(2), 21-81 (2013).

3. S. Ismadji, F.Soetaredjo, A. Ayucitra. Clay Materials for Environmental Remediation, Springer, 1, (2015). 
4. S. Xu, G. Sheng, \& S. A. Boyd. Use of organoclays in pollution abatement. Advances in agronomy (USA) (1997).

5. D. M. Moore, \& R. C. Reynolds. X-ray Diffraction and the Identification and Analysis of Clay Minerals: Oxford University Press (1997).

6. M. Chaves, \& M. Oliveira. Mechanisms underlying plant resilience to water deficits: prospects for water-saving agriculture. J. exp. Bot., 55(407), 2365-2384 (2004).

7. S. Guerra, L. Merat, R. San Gil \& L. Dieguez. Alkylation of benzene with olefins in the presence of zirconium-pillared clays. Catal. Today., 133, 223-230 (2008).

8. M. Toor, B. Jin, S. Dai, \& V. Vimonses. Activating natural bentonite as a cost-effective adsorbent for removal of Congo-red in wastewater. J. Ind. Eng. Chem., 21, 653-661 (2015).

9. P. Komadel, \& J. Madejová. Acid activation of clany minerals. Handbook of Clay Science, 385-408 (2013).

10. P. A. O'Day, \& D. Vlassopoulos. Mineralbased amendments for remediation. Elements (Quebec, Quebec), 6(6), 375 (2010).

11. A. A. Zagorodni. Ion exchange materials: properties and applications: properties and applications: Elsevier, 1, (2006).

12. G. Yuan, B. Theng, G. Churchman, \& W. Gates. Clays and clay minerals for pollution control. Handbook of Clay Science, 1, 587-644 (2013).

13. C. T. Chiou. Partition and adsorption of organic contaminants in environmental systems: John Wiley \& Sons (2003).

14. M. Gräfe, M. Nachtegaal, \& D. L. Sparks. Formation of metal-arsenate precipitates at the goethite-water interface. Environ. Sci. tech., 38(24), 6561-6570 (2004).

15. G. E. Brown, \& G. Calas. Environmental mineralogy-understanding element behavior in ecosystems. Comptes Rendus Geoscience, 343(2), 90-11 (2011).

16. T. Anirudhan, \& M. Ramachandran. Adsorptive removal of tannin from aqueous solutions by cationic surfactant-modified bentonite clay. J. Colloid Interface Sci., 299(1), 116-124 (2006).

17. S. Bedin, M. Oliveira, M. Vieira, M. Silva. \& O. Santos. Adsorption of toluene in batch system in natural clay and organoclay. Chem. Eng. Trans, 32, 313-318 2013).

18. J. A. Smith, P. R. Jaffe, \& C. T. Chiou. Effect of ten quaternary ammonium cations on tetrachloromethane sorption to clay from water. Environ.1 sci. tech., 24(8), 1167-117 (1990).

19. W. Jaynes, \& G. Vance. Sorption of benzene, toluene, ethylbenzene, and xylene (BTEX) compounds by hectorite clays exchanged with aromatic organic cations. Clays Clay Miner., 47(3), 358-365 (1999).
20. C. Bertagnolli, \& M. Silva. Characterization of Brazilian Bentonite Organoclays as sorbents of petroleum-derived fuels. Mater. Res., 15(2), 253-259 (2012).

21. Koh, S.-M., \& Dixon, J. B. Preparation and application of organo-minerals as sorbents of phenol, benzene and toluene. Appl. Clay Sci., 18(3-4), 111-122 (2001).

22. W. Jaynes, \& S. Boyd. Hydrophobicity of siloxane surfaces in smectites as revealed by aromatic hydrocarbon adsorption from water. Clay Miner., 39(4), 428-436 (1991).

23. M. Carvalho, M. Da Motta, M. Benachour, D. Sales, \& C. Abreu. Evaluation of BTEX and phenol removal from aqueous solution by multi-solute adsorption onto smectite organoclay. J. Hazard. Mater., 239, 95-101 (2012).

24. F. Qu, L. Zhu, \& K. Yang. Adsorption behaviors of volatile organic compounds (VOCs) on porous clay heterostructures ( $\mathrm{PCH})$. J. Hazard. Mater., 170(1), 7-12 (2009).

25. H. Nourmoradi, M. Nikaeen, \& Khiadani M. Removal of benzene, toluene, ethylbenzene and xylene (BTEX) from aqueous solutions by montmorillonite modified with nonionic surfactant: Equilibrium, kinetic and thermodynamic study. Chem. Eng. J., 191, 341348 (2012).

26. S. Noreen, H. Bhatti, N. Nousheen, Sadaf. H. Batch and fixed bed adsorption study for removal of Drimarine Black CL-B dye from aqeous solution using a lignocellulosic waste: a cost effective adsorbent. Ind. Corps Produc, 50, 568-579.

27. K. Shroff, V. Vaidya. Kinetics and equilibrium studies on biosorption of nickel from aqeous solution by dead fungal biomass of Mucar heimalis. Chem. Eng. J. 171, 1234-1245 (2011).

28. I. Ullah, R. Nadeem, M. Iqbal, Q. Manzoor. Biosorption of chromimum onto native and immobilized sugarcane baggase waste biomass. Ecol. Eng. 60, 99-107 (2013).

29. M. Iqbal, M. Abbas, M. Arshad, T. Hussain, A. U. Khan, N. Masood, M.A.Tahir. Gamma radiation treatment for reducing cytotoxicity and mutagenicity in industrial wastewater. Polish J. Environ. Stud. 24, 2745-2750 (2015).

30. P.D Saha, A. Dey, P. Marik. Batch removal of chromium(VI) from aqueous solutions using wheat shell as adsorbent: process optimization using response surface methodology. Desalin. Water Treat. 39, 95-102 (2015).

31. G. Morozov, V. Breus, S. Nekludov \& I. Breus. Sorption of volatile organic compounds and their mixtures on montmorillonite at different humidity. Colloids Surf. A. Physicochem. Eng. Asp., 454, 159-171 (2014).

32. G. Alther. Using organoclays to enhance carbon filtration. Waste Management, 22(5), 507-513 (2002). 